\title{
Scaling rules in optomechanical semiconductor micropillars
}

\author{
S. Anguiano, ${ }^{1}$ P. Sesin, ${ }^{1}$ A. E. Bruchhausen, ${ }^{1}$ F. R. Lamberti, ${ }^{2}$ I. Favero, ${ }^{3}$ M. Esmann, ${ }^{2}$ I. Sagnes, ${ }^{2}$ A. Lemaître,${ }^{2}$ \\ N. D. Lanzillotti-Kimura, ${ }^{2}$ P. Senellart, ${ }^{2, *}$ and A. Fainstein ${ }^{1, \dagger}$ \\ ${ }^{1}$ Centro Atómico Bariloche and Instituto Balseiro, CNEA, CONICET, 8400 San Carlos de Bariloche, Rio Negro, Argentina \\ ${ }^{2}$ Centre de Nanosciences et de Nanotechnologies, CNRS, Université Paris-Sud, Université Paris-Saclay, C2N, \\ Avenue de la Vauve, 91120 Palaiseau, France \\ ${ }^{3}$ Matériaux et Phénomènes Quantiques, Université Paris Diderot, CNRS, UMR No. 7162, Sorbonne Paris Cité, \\ 10 Rue Alice Domon et Léonie Duquet, 75013 Paris, France
}

(Received 8 August 2018; published 6 December 2018)

\begin{abstract}
Semiconductor pillar microcavities have recently emerged as a promising optomechanical platform in the unprecedented 20-GHz frequency range. Currently established models for the mechanical behavior of micropillars, however, rely on complete numerical simulations or semianalytical approaches, which makes their application to experiments notoriously difficult. Here we overcome this challenge with an effective model by reducing the full, hybridized mechanical mode picture of a micropillar to an approach that captures the observed global trends. We show experimentally the validity of this approach by studying the lateral size dependence of the frequency, amplitude, and lifetime of the mechanical modes of square-section pillar microcavities, using room-temperature pump-probe microscopy. General scaling rules for these quantities are found and explained through simple phenomenological models of the physical phenomena involved. We show that the energy shift $\Delta \omega_{m}$ of the modes due to confinement is dependent on the inverse of their frequency $\omega_{0}$ and lateral size $L\left(\Delta \omega_{m} \propto 1 / \omega_{0} L^{2}\right)$ and that the mode lifetime $\tau$ is linear with pillar size and inversely proportional to their frequency $\left(\tau \propto L / \omega_{0}\right.$ ). The mode amplitude is in turn inversely proportional to the lateral size of the considered resonators. This is related to the dependence of the optomechanical coupling rate $\left(g_{0} \propto 1 / L\right)$ with the spatial extent of the confined electromagnetic and mechanical fields. Using a numerical model based on the finite-element method, we determine the magnitude and size dependence of $g_{0}$ and, by combining the results with the experimental data, we discuss the attainable single-photon cooperativity in these systems. The effective models proposed and the scaling rules found constitute an important tool in micropillar optomechanics and in the future development of more complex micropillar based devices.
\end{abstract}

DOI: 10.1103/PhysRevA.98.063810

\section{MOTIVATION}

A variety of optomechanical resonators able to reach increasingly higher frequencies are at the center of intense activity [1-3]. The underlying motivation is the development of optomechanical platforms that could be easily prepared and manipulated in their quantum-mechanical state of motion, at higher experimental temperatures [4]. Additionally, the realization of high-frequency optomechanical systems is highly desirable for metrology applications [5] and for ultrafast information processing [6,7]. As part of this pursuit, micropillar resonators operating at ultrahigh frequencies (greater than $10 \mathrm{GHz}$ ) and presenting strong optomechanical interactions and state-of-the-art $Q$-frequency products have been reported recently $[8,9]$. This frequency range, however, comes together with critical challenges. For instance, mechanical noise spectral measurements, widely used in the context of cavity optomechanics experiments, are here challenging to implement, due to the high frequency of the probed mechanical

\footnotetext{
*pascale.senellart@lpn.cnrs.fr

${ }^{\dagger}$ Author to whom correspondence should be addressed: afains@cab.cnea.gov.ar
}

modes. Therefore, alternative techniques need to be devised to study the vibrational spectra and dynamics with the required bandwidth and resolution [10].

An optomechanical system can be described by several parameters, related to both the optical and mechanical components, as well as to the strength of their interaction [11]. Namely, for one such system to be efficient, high mechanical and optical quality factors $\left(Q_{m}\right.$ and $\left.Q_{\text {opt }}\right)$, aside from a high optomechanical coupling rate $\left(g_{0}\right)$, are required. A high value of $\frac{g_{0}}{\kappa}$ (with $\kappa=\frac{\omega_{\text {opt }}}{Q_{\text {opt }}}$ the optical damping) is favorable for nonlinear quantum-optomechanical experiments working with single photons and phonons $[12,13]$. The singlephoton cooperativity $C_{0}=\frac{4 g_{0}^{2}}{\kappa \Gamma_{m}}$ (with $\Gamma_{m}=\frac{\omega_{m}}{Q_{m}}$ the mechanical damping), on the other hand, is relevant for phenomena such as optomechanically induced transparency [14] and to attain the strong-coupling regime [15]. It is the purpose of this paper to study the mechanical frequencies, quality factors, and the magnitude of the optomechanical coupling of micropillar resonators and how these parameters vary as a function of lateral confinement. Experimental results on pump-probe spectroscopy are presented, together with model calculations and a discussion of the physics involved. The present limitations and prospects for improvement are also addressed. 


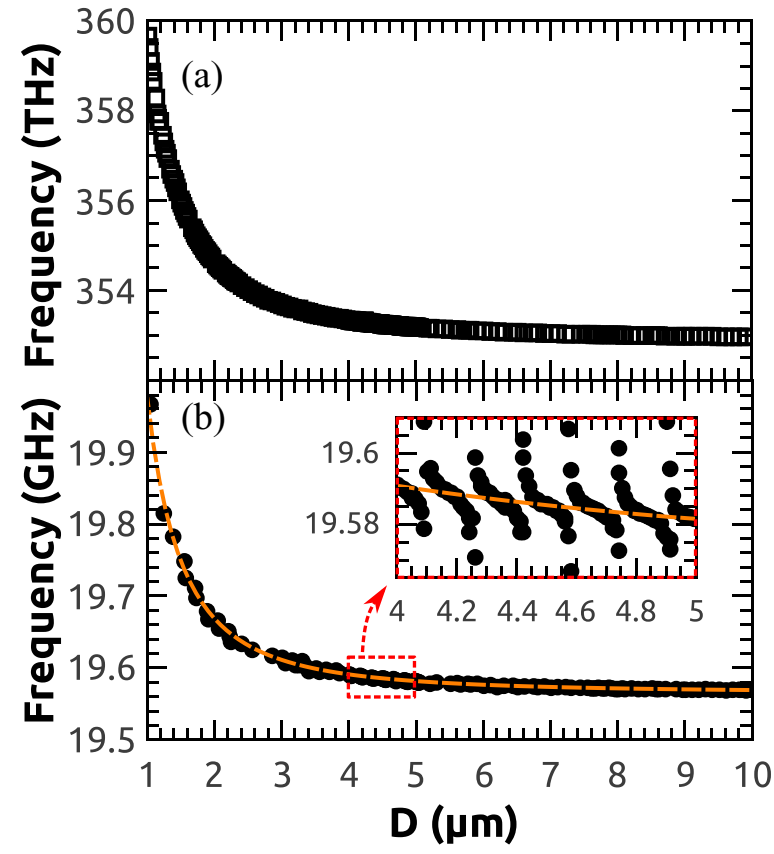

FIG. 1. (a) Optical cavity mode frequencies as a function of pillar diameter $D$. (b) Acoustic cavity mode frequency as a function of pillar diameter. In the inset we highlight the avoided-crossing behavior of the mechanical modes. The dashed orange line is a numerical fit using Eq. (2), with $v_{s}=5285 \mathrm{~m} / \mathrm{s}$.

\section{SIMPLIFIED MODEL}

It has been shown recently that the vibrational dynamics of distributed Bragg reflector (DBR) pillar resonators have a certain degree of complexity due to the mixing of radial and $z$-polarized breathing modes, which become coupled due to the Poisson ratio [9]. This leads to strong variations of the mechanical quality factor $Q_{m}$ and $g_{0}$ depending on the relative weight of the breathing (higher- $Q_{m}$ ) and radial (lower- $Q_{m}$ ) components. Finite-element calculations were performed for both the fundamental acoustic and optical cavity modes, varying the pillar size. The calculations were performed for a pillar $\lambda / 2$ bulk-GaAs cavity enclosed by two DBRs consisting of alternating $\mathrm{Ga}_{0.9} \mathrm{Al}_{0.1} \mathrm{As} / \mathrm{Ga}_{0.05} \mathrm{Al}_{0.95} \mathrm{As} \lambda / 4$ layers, 28 pairs at the bottom and 24 on top. The resulting frequency dependence is presented in Fig. 1. A cylindrical rather than a square geometry was used in all cases, for computational efficiency. The optical response [Fig. 1(a)] shows a smooth dependence with diameter, while the mechanical response [Fig. 1(b)] presents a complex avoided-crossing behavior, highlighted in the inset in Fig. 1 [9]. Globally, however, it is observed that the diameter dependence of the mechanical frequencies is quite similar to that of the optical mode. Only upon close inspection are these anticrossings revealed. Moreover, the spatial distribution of a predominantly z-polarized acoustic mode, shown in Fig. 2, resembles that expected for the optical case [16].

Based on the above considerations, the acoustic cavity modes in a micropillar can be described in an approximate way by considering zero displacement on the lateral surfaces and neglecting the anticrossing behavior. As a result, within this simplified approach, we obtain fully $z$-polarized modes with a lateral distribution analogous to the case of a vibrating

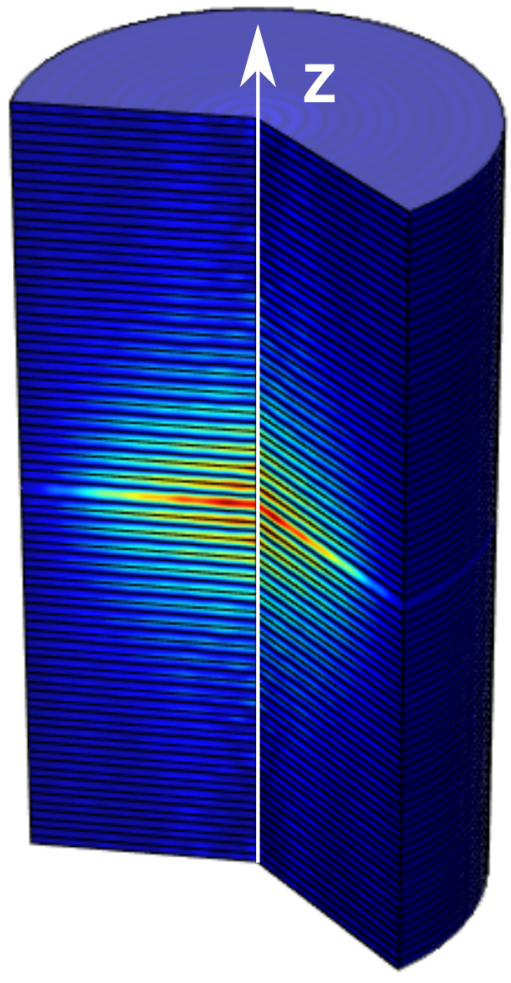

FIG. 2. Finite-element simulation showing the absolute value of the volumetric strain due to the first acoustic cavity mode $(\sim 19 \mathrm{GHz})$ for a 3- $\mu$ m-diam micropillar.

membrane (drumlike modes). To do so, we start with the general case of the angular dispersion of a mechanical cavity mode in a planar sample

$$
q^{2}=q_{0}^{2}+q_{\|}^{2}, \quad \omega_{m}^{2}=\omega_{0}^{2}+v_{s}^{2} q_{\|}^{2},
$$

where $q_{0}$ and $q_{\|}$are the phonons' $z$ and in-plane components of the wave vector $q$, respectively, $v_{s}$ is an effective sound speed of the sample, and $\omega_{0}$ is the angular frequency of the $q_{\|}=0$ mode. Replacing the in-plane wave vector with the one associated with the confined modes of a circular section pillar, the angular frequencies of the confined modes are approximately given by [16]

$$
\omega_{m}^{2}=\omega_{0}^{2}+\left(\frac{2 v_{s}}{D}\right)^{2} X_{b l}^{2}
$$

where $D$ is the pillar diameter and $X_{b l}$ the $b$ th root of the $l$ th-order Bessel function. For a square-section pillar Eq. (1) can be approximated by $[17,18]$

$$
\omega_{m}^{2}=\omega_{0}^{2}+\left(\frac{\pi v_{s}}{L}\right)^{2}\left[\left(\eta_{x}+1\right)^{2}+\left(\eta_{y}+1\right)^{2}\right],
$$

with $L$ the lateral size and $\eta_{v} \in \mathbb{N}_{0}$. Essentially we are considering that the lateral wave vector $q_{\|}$can take some discrete values, given by sinusoidal (Bessel) in-plane distributions for square-section (circular-section) pillars. Using Eq. (2) to fit the finite-element results shown in Fig. 1(b), we get $v_{s}=$ $5285 \mathrm{~m} / \mathrm{s}$, which is an intermediate value between the sound speeds for $\mathrm{Ga}_{0.9} \mathrm{Al}_{0.1}$ As $(4800 \mathrm{~m} / \mathrm{s})$ and $\mathrm{Ga}_{0.05} \mathrm{Al}_{0.95} \mathrm{As}(5593$ $\mathrm{m} / \mathrm{s}$ ), as expected for the structure under study. The fit of the more complex finite-element results describes very well the 


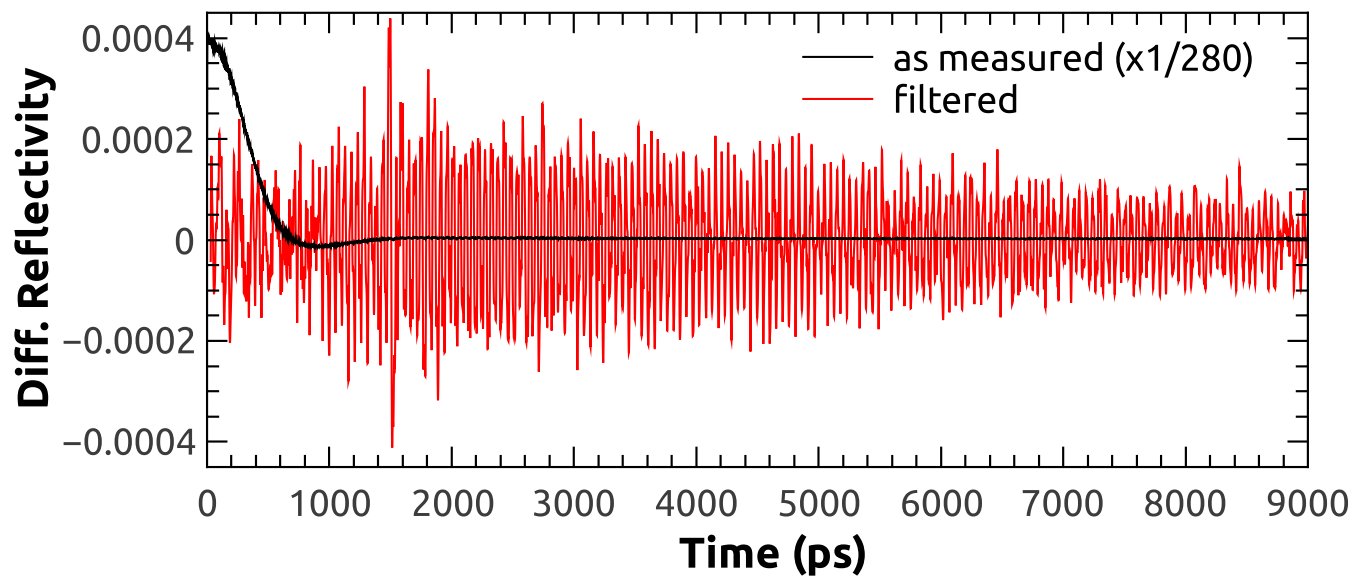

FIG. 3. Typical time-resolved differential reflectivity trace. The black line corresponds to the as-measured trace, while the red one has been Fourier filtered in order to leave only the oscillatory components, with frequencies ranging from 5 to $100 \mathrm{GHz}$.

general trend of the confined mechanical modes and thus will be used as a basic phenomenological model in what follows.

\section{LATERAL CONFINEMENT FREQUENCY SHIFT}

The samples studied in this work consist of square-section micropillars with lateral size ranging from 2 to $60 \mu \mathrm{m}$, which were dry etched from a $\lambda / 2$ bulk-GaAs planar cavity enclosed by two DBRs, grown by molecular beam epitaxy. The DBRs consist of alternating $\mathrm{Ga}_{0.9} \mathrm{Al}_{0.1} \mathrm{As} / \mathrm{Ga}_{0.05} \mathrm{Al}_{0.95} \mathrm{As} \lambda / 4$ layers, 28 pairs at the bottom and 24 on top.

We implemented a time-resolved differential optical reflectivity (pump-probe) measurement [19,20] with micrometer lateral resolution. All the presented experiments were performed at room temperature and with the laser wavelength set around $882 \mathrm{~nm}$. In these experiments, two pulses of different intensity and orthogonal polarization are focused on the same spot. The pump ( $5 \mathrm{~mW})$ is used to impulsively generate a coherent population of acoustic phonons in the sample (it also generates an increase of the pillars mean temperature of $\sim 1.5 \mathrm{~K}$, which nonetheless does not seem to affect their mechanical properties, within our experimental resolution), while the probe $(0.5 \mathrm{~mW})$, delayed from the former, is used to measure the phonon-induced perturbation in the optical reflectivity. By changing the delay between pump and probe pulses, the temporal evolution of the optical reflectivity can be reconstructed. To do this, the reflected probe is separated from the pump beam, using a polarizing beam splitter, and is guided to a photodiode, where the differential reflectivity is measured with a lock-in amplifier. A typical time-resolved differential reflectivity trace is presented in Fig. 3. The black line corresponds to the as-measured trace, while the red one has been filtered in order to leave only the oscillatory components ranging from 5 to $100 \mathrm{GHz}$. If a Fourier transform is applied to this trace, a vibrational spectrum like the one shown in Fig. 4 is obtained. Two sharp peaks are distinguished, corresponding to the first two acoustic cavity modes. The insets show a close-up of the $\sim 19$ - and $\sim 57-\mathrm{GHz}$ modes for four pillars of different lateral size. The vertical dashed lines are guides to the eye that mark the central frequency of each acoustic mode for the larger pillar. A shift to higher frequencies is seen for the 3 - and $2-\mu \mathrm{m}$ pillars, particularly for the $\sim 19-\mathrm{GHz}$ mode.
The observed shift can be attributed to the lateral confinement of the acoustic field. To address this, we will make a few theoretical considerations first.

The dashed curves in the insets of Fig. 4 are the results of simulating the spectra including for each pillar modes of different in-plane order $\eta_{\nu}$, using Eq. (3) to calculate the frequencies, and using experimental values for the phonon lifetimes. Note the excellent agreement between model and experiments, concerning the modes frequencies. The lifetimes were extracted directly from the temporal traces (like the one shown in Fig. 3) by fitting the amplitude time dependence of each acoustic mode with an exponential decay. The amplitudes used for the acoustic modes with different in-plane order are obtained from the overlap integral between the field associated with the fundamental optical mode and the strain associated with each of these orders (see further below). A difference in the FWHM for the bigger pillars is evident between the experimental and the simulated curves. The reason for this difference comes from the fact that the experimental Fourier transforms are resolution limited by the length of the

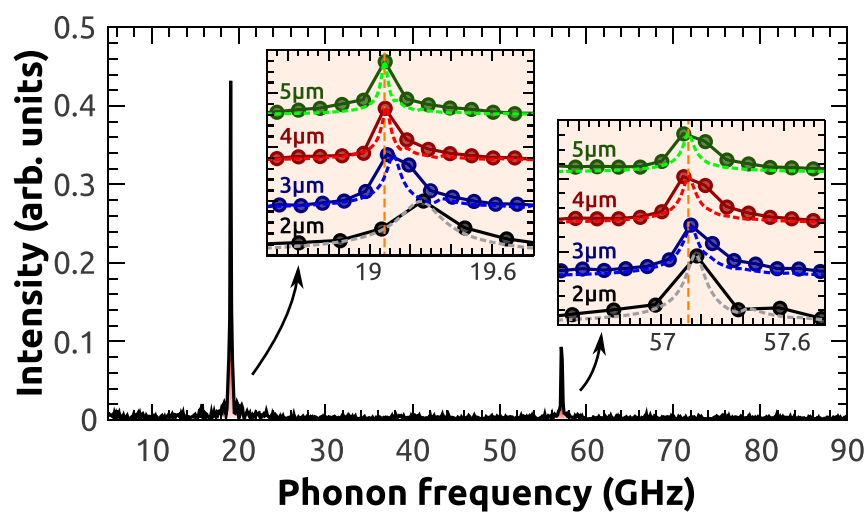

FIG. 4. Typical vibrational spectra showing the first two acoustic cavity modes of the structure. The two insets show a close-up of the modes of pillars with lateral size ranging from 2 to $5 \mu \mathrm{m}$. Measurements are presented with solid lines and circles, while the theoretical results are presented with dashed lines. The vertical orange dashed lines are guides to the eye marking the center of the $5-\mu \mathrm{m}$ pillar modes. 


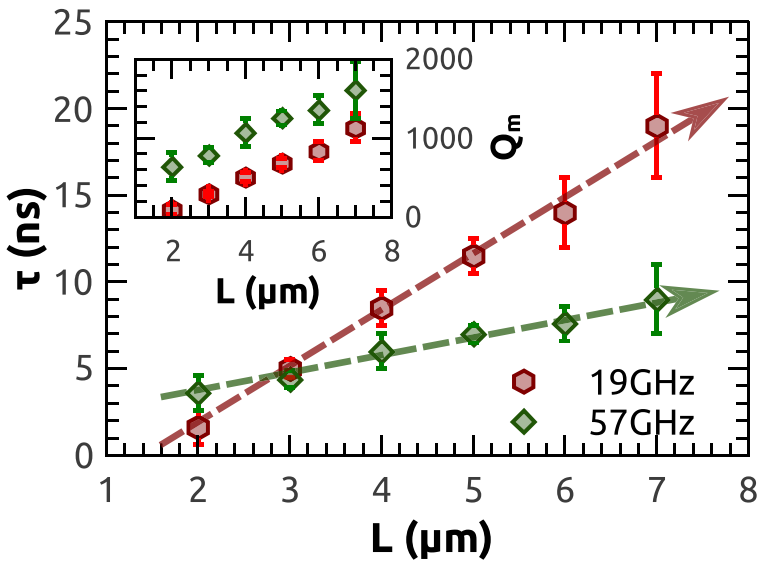

FIG. 5. Acoustic mode lifetime $\tau$ as a function of the lateral size $L$. The dashed lines correspond to linear fits. The inset shows the dependence of the corresponding mechanical quality factor $Q_{m}$ on lateral size.

temporal scan, which for the bigger pillars is shorter than the phonon lifetimes. For the $2-\mu \mathrm{m}$ pillar, instead, the scan time is relatively longer in comparison with the shorter lifetimes of its acoustic modes; therefore, the agreement is better.

Note the marked difference in Fig. 4 between the shift due to confinement of the $\sim 19-\mathrm{GHz}$ mode versus the $\sim 57-\mathrm{GHz}$ one. It is evident that the former is larger than the latter. This is well reproduced by the model and can be understood by expanding Eq. (3) in a Taylor series

$$
\omega_{m} \approx \omega_{0}+\frac{1}{2 \omega_{0}}\left(\frac{\pi v_{s}}{L}\right)^{2}\left[\left(\eta_{x}+1\right)^{2}+\left(\eta_{y}+1\right)^{2}\right] .
$$

Here an inverse dependence of the shift with frequency and with the square of the pillar lateral size is obtained $\left(\omega_{m}-\right.$ $\omega_{0}=\Delta \omega_{m} \propto 1 / \omega_{0} L^{2}$ ). Therefore, modes corresponding to higher order of confinement along $z$ (with higher angular frequency $\omega_{0}$ ) will be less affected by lateral confinement. In fact, the experimental difference in shift between the $\sim 19$ and $\sim 57-\mathrm{GHz}$ modes is $\sim 3$, in agreement with this simple prediction.

\section{MECHANICAL MODE LIFETIME}

We address next the acoustic mode lifetimes $\tau$. In Fig. 5 we present its dependence with lateral size for the $\sim 19-\mathrm{GHz}$ and $\sim 57-\mathrm{GHz}$ mechanical cavity modes. As was already mentioned, these values are obtained by fitting the temporal dependence of the amplitude of the modes with exponential decays. We have extracted the lifetimes only for pillars up to $7 \mu \mathrm{m}$ because for larger pillars the photocarrier recombination time is so long that the useful temporal window (i.e., not affected by the carrier-induced cavity-mode dynamics) does not allow a good fit (a more detailed discussion on the procedure followed can be found in the Supplemental Material in Ref. [8]). This is also the reason why the error in $\tau$ in Fig. 5 increases with increasing $L$. The experimental data show a linear dependence of $\tau$ with $L$. The slope for the $\sim 19-\mathrm{GHz}$ mode is $\sim 2.9$ times larger than that for the $\sim 57-\mathrm{GHz}$ mode, i.e., experimentally it is observed that $\tau \approx \alpha \frac{L}{\omega_{0}}+\beta$. The inset in Fig. 5 shows the estimated room-temperature mechanical quality factor $Q_{m}=\frac{\omega_{m} \tau}{2}$ (the factor 2 appears because $\tau$ in our case corresponds to the decay rate of the mechanical mode amplitude, instead of the intensity) as a function of pillar size. The values obtained for $Q_{m}$ are somewhat smaller than the optical quality factor of these structures $\left(Q_{\text {opt }} \approx\right.$ $10000)$. Since the refractive index mismatch and the acoustic impedance mismatch are almost identical in the $\mathrm{Al}_{x} \mathrm{Ga}_{(x-1)} \mathrm{As}$ alloys [21], optical and mechanical losses through the bottom DBR and towards the substrate should be similar and thus the nominal value of mechanical and optical $Q$ factors should be of the same order. The fact that they are not is an indication that surface defects might be more relevant to mechanical than to optical losses.

When decreasing the lateral size of a pillar, besides confining the mechanical modes, the free surfaces start to play an important role. In particular, these surfaces can present a higher concentration of crystallographic defects (compared to the interfaces defined by the molecular beam epitaxy growth) due to the fabrication process or to superficial oxidation. For high-frequency phonons, these defects act as preferential scattering points.

Consider the case of a plane wave interacting with a rough surface. Each wavefront is defined as a plane of constant phase. After interaction with this surface, the reflected wave will have a new distribution, which will deviate from the original in proportion to the degree of roughness of the surface. Since part of the wave will retain its former coherence, it is possible to define an effective reflection coefficient, analogous to the case of a plane wave interacting with a perfectly flat surface [22-24]

$$
r=e^{-4 \sigma^{2} \omega_{0}^{2} / v_{s}^{2}}
$$

with $\sigma$ the standard deviation of the roughness distribution of the surface.

Let us now consider a wavefront inside a pillar of lateral size $L$. This wavefront will have, as we already mentioned, a nonzero in-plane wave-vector component. The coherent acoustic waves created inside a pillar will be reflected on the free surfaces $N$ times after a given time $t$, with

$$
N=\frac{v_{\|}}{L} t
$$

where $v_{\|}$is the effective in-plane speed of the considered mode of angular frequency $\omega_{0}$, given by $v_{\|}=\frac{\partial \omega_{m}}{\partial q_{\|}}$. We have already introduced an approximation to the dispersion relation in Eq. (4). Rewriting it explicitly as a function of $q_{\|}$, we get

$$
\omega_{m} \approx \omega_{0}+\frac{v_{s}^{2}}{2 \omega_{0}} q_{\|}^{2} .
$$

Thus, the effective in-plane speed of a wavefront corresponding to a confined mechanical mode is inversely proportional to the frequency, i.e., $v_{\|} \approx \frac{v_{s}^{2}}{\omega_{0}} q_{\|}$. Then we can rewrite Eq. (6) as

$$
N \approx \frac{v_{s}^{2} q_{\|}}{\omega_{0} L} t
$$




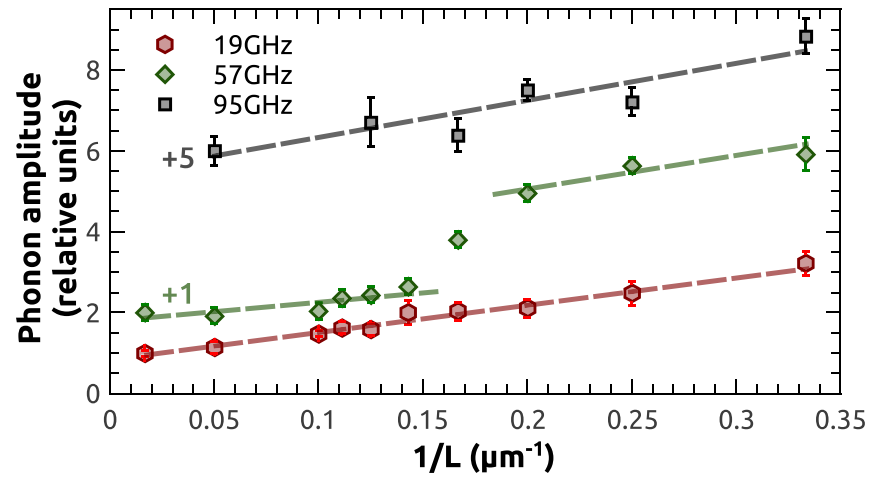

FIG. 6. Acoustic mode amplitude as a function of the inverse of the lateral size $1 / L$ for the first three cavity modes. The amplitudes of the 19- and $57-\mathrm{GHz}(95-\mathrm{GHz})$ modes are normalized by the amplitude corresponding to the $60-\mu \mathrm{m}(20-\mu \mathrm{m})$ pillar and are vertically shifted for clarity. The dashed lines correspond to linear fits.

The amplitude $u$ of the wavefront will depend consequently on time in the following way:

$$
u(t)=u_{x}(t) u_{y}(t)=u_{0} r^{2 N}=u_{0} e^{-8 \sigma^{2} q_{\|}\left(\omega_{0} / L\right) t} .
$$

Thus, assuming an exponential dependence of $u(t)$ with a certain lifetime $\tau$, i.e., $u(t)=u_{0} e^{-t / \tau}$, it follows that

$$
\tau \propto \frac{L}{\omega_{0}} .
$$

This consistently explains the linear dependence and the different slopes observed in Fig. 5. Furthermore, using the measured dependences for the lifetimes, it is possible to estimate for the surface roughness a value of $\sigma \approx 16 \mathrm{~nm}$. This is consistent with the oxidation-induced damage of the Al-rich layers of the structure.

\section{MODE AMPLITUDE}

In addition to the mechanical mode lifetime, the amplitude of the vibrations also shows a strong dependence on pillar size. In Fig. 6 we present the dependence of this amplitude on the inverse of the lateral size for the first three mechanical modes. We observe that, for all the probed modes, maximal optomechanical interaction strengths are obtained for the micropillars with the smallest lateral dimensions. An approximate linear dependence with similar slope is evident for the $\sim 19$ - and $\sim 95-\mathrm{GHz}$ modes, while the $\sim 57-\mathrm{GHz}$ mode shows a somewhat different behavior, with two distinct zones, each one with a slope similar to the other modes. Since photoelasticity is the dominant detection mechanism [21], we can estimate the detection function $\mathcal{D}$ by the overlap integral $[25,26]$

$$
\mathcal{D} \propto \int_{V} p_{12}(z) \frac{\partial U(x, y, z)}{\partial z}|E(x, y, z)|^{2} d V .
$$

Here $p_{12}(z)$ is the photoelastic constant in the different materials, $E(x, y, z)$ the probe electric field distribution (scalar), and $U(x, y, z)$ the acoustic mode displacement field (scalar). Equation (11) is taken in scalar form because, due to the mainly z-polarized character of the vibrations, the component of the photoelastic tensor that has the strongest influence in these structures is $p_{12}$ and the rest can therefore be ignored [27]. This expression was already used to calculate the mechanical spectra as expected from a pump and probe experiment in Fig. 4. It allows us to estimate the influence that each mechanical mode has on the optical response of the microcavities. For the laser wavelength used we will consider $p_{12} \neq 0$ only in the spacer (of thickness $d$ ) since it is known that the photoelastic tensor is strongly resonant [27] and the laser wavelength is very close to the GaAs gap at room temperature $(\sim 870 \mathrm{~nm})$ but far from those of the alloys present in the DBRs $(\sim 800$ and $\sim 580 \mathrm{~nm})$.

Let the fields have a spatial distribution given by

$$
\begin{aligned}
& E(x, y, z)=E_{0} e(x, y, z), \\
& U(x, y, z)=u_{0} u(x, y, z),
\end{aligned}
$$

where $E_{0}\left(u_{0}\right)$ is the maximum electric (displacement) field amplitude. Then the detection function can be rewritten as

$\mathcal{D} \propto p_{12} u_{0}\left|E_{0}\right|^{2} \int_{0}^{L} \int_{0}^{L} \int_{0}^{d} \frac{\partial u(x, y, z)}{\partial z}|e(x, y, z)|^{2} d x d y d z$,

$$
\begin{aligned}
\mathcal{D} \propto & p_{12} u_{0}\left|E_{0}\right|^{2} L^{2} d \int_{0}^{1} \int_{0}^{1} \int_{0}^{1} \frac{\partial u(q, v, w)}{\partial w} \\
& \times|e(q, v, w)|^{2} d q d v d w .
\end{aligned}
$$

The integrals in Eq. (14b) are adimensional, and since the functional dependence of the distribution of the fields is not dependent on the size, these integrals must be constant. Therefore, we get

$$
\mathcal{D} \propto u_{0}\left|E_{0}\right|^{2} L^{2} .
$$

Assuming optimal coupling in all cases so that the number of photons in the cavity does not depend on the pillar size, from energy considerations [28] the maximum amplitude of the electromagnetic field $E_{0}$ associated with the optical cavity mode can be considered to depend on the volume $V$ of the sample as

$$
E_{0} \propto \frac{1}{\sqrt{V}} .
$$

The same energy-conservation arguments apply for the mechanical equations and we can thus consider that the acoustic field amplitude depends on confinement in the same way as the electromagnetic field

$$
u_{0} \propto \frac{1}{\sqrt{V}} .
$$

Summing up, we get

$$
\mathcal{D} \propto \frac{L^{2}}{V^{3 / 2}}=\frac{1}{H_{\mathrm{eff}}^{3 / 2} L},
$$

with $H_{\text {eff }}$ the effective vertical confinement length of the pillar cavity mode. Since $H_{\text {eff }}$ is constant with the lateral dimensions of the considered structure, we get that $\mathcal{D} \propto 1 / L$, coincident with the experimental data presented in Fig. 6.

There is a detail, however, regarding the $\sim 57-\mathrm{GHz}$ mode that we are unable to describe with this model. It is evident 
in Fig. 6 that the $\sim 19$ - and $\sim 95-\mathrm{GHz}$ modes fall almost perfectly on the linear fit and have a similar slope, while the $\sim 57-\mathrm{GHz}$ mode does not. There seems to be a progressive change around $L \approx 6 \mu \mathrm{m}$, which shows that the data below and above this point are similarly dependent on $L$ like the two other modes. Though we presently do not have a definitive explanation for this behavior, we speculate that this mode in particular may suffer a distributional change, related to the anticrossings previously discussed, which could affect the optomechanical coupling [9]. More experiments are required to satisfactorily explain this particular feature.

\section{OPTOMECHANICAL COUPLING}

Resuming the dependence of $\mathcal{D}$ on $L$ obtained with the simple model proposed, note that the integral in Eq. (11) represents the interaction strength between the confined optical and mechanical modes, mediated by the photoelastic effect. In fact, this same integral is related to the vacuum optomechanical photoelastic coupling factor $g_{0}^{\mathrm{ph}}[21,29]$, which is associated with the electrostrictive optical forces exerted by the photons trapped inside the resonator. It is thus interesting to calculate $g_{0}^{\mathrm{ph}}$ as a function of the lateral size and compare it with the experimental results. We do this next, based on finiteelement modeling (COMSOL). Also, for completeness, we will compare the photoelastic coupling with the other mechanism involved in the optomechanical response of the system studied in this work. Such interaction is mediated by the motion (associated with the confined acoustic mode displacement field) of the boundaries between the different semiconductor layers inside the micropillar resonator. The related coupling constant is known as the geometric coupling factor $g_{0}^{\text {geo }}$ and is related to the radiation pressure forces [21,29]. Overall, the total optomechanical coupling factor of a given system reads

$$
g_{0}=-\frac{d \omega_{\mathrm{opt}}}{d u} x_{\mathrm{ZPF}}=-\frac{d \omega_{\mathrm{opt}}}{d u} \sqrt{\frac{\hbar}{2 m_{\mathrm{eff}} \omega_{m}}},
$$

where $\frac{d \omega_{\text {opt }}}{d u}$ indicates the change in the optical resonance frequency due to a differential mechanical displacement (which takes into account both photoelastic and geometrical coupling), $x_{\mathrm{ZPF}}$ is the zero-point motion of the oscillator, and $m_{\text {eff }}$ is its effective mass. The optomechanical coupling $g_{0}$ measures the frequency shift of the optical mode $\omega_{\text {opt }}$ imparted by $x_{\mathrm{ZPF}}$. The effective mass can be calculated by considering that the potential energy of this parametrized oscillator has to be equal to the potential energy stored inside the micropillar resonator

$$
\frac{1}{2} \omega_{m}^{2} \int \rho(\vec{r})|\vec{U}(\vec{r})|^{2} d \vec{r}=\frac{1}{2} m_{\mathrm{eff}} \omega_{m}^{2} u_{0}^{2},
$$

where $\rho(\vec{r})$ is the mass density distribution. The confined mode displacement field is represented by a vector field $\vec{U}(\vec{r})$. For a unit-normalized mode displacement $\vec{u}(\vec{r})$, the motion of the mode can be parametrized as $\vec{U}(\vec{r}) \rightarrow \vec{U}\left(u_{0}, \vec{r}\right)=$ $u_{0} \vec{u}(\vec{r})$, with $u_{0}$ the maximum displacement present in the sample. The chosen normalization implies the following definition of the effective mass:

$$
m_{\mathrm{eff}}=\int \rho(\vec{r})|\vec{u}(\vec{r})|^{2} d \vec{r} .
$$

To understand the overall size dependence of the optomechanical coupling factors, we focus on the z-polarized modes, avoiding the anticrossing regions illustrated in Fig. 1(b).

To compute the geometric contribution we follow the analysis by Johnson et al. [30]. In Refs. [1,29] this approach is used to evaluate the optomechanical coupling in microdisk resonators. In that system, there is just one interface between dielectrics (between GaAs and air). For the DBR microcavities described here, we have to compute the contributions due to the multiple boundaries, given by

$$
g_{0}^{\mathrm{geo}}=\frac{\omega_{\mathrm{opt}}}{2} \sum_{i} \frac{\oint_{A_{i}}(\vec{u} \cdot \hat{n})\left[\Delta \epsilon_{i}\left|\vec{E}_{\|}\right|^{2}-\Delta\left(\epsilon_{i}^{-1}\right)\left|\vec{D}_{\perp}\right|^{2}\right] d A_{i}}{\int \epsilon|\vec{E}|^{2} d V} x_{\mathrm{ZPF}}
$$

where $\vec{u}$ is the normalized displacement field, $\hat{n}$ is the unitary normal-surface vector corresponding to the interface, $\Delta \epsilon_{i}=$ $\epsilon_{i, \text { left }}-\epsilon_{i, \text { right }}, \Delta \epsilon_{i}^{-1}=\epsilon_{i, \text { left }}^{-1}-\epsilon_{i, \text { right }}^{-1}, \vec{E}_{\|}$is the electric field component parallel to the interface surface, and $\vec{D}_{\perp}$ is the displacement field component normal to the interface surface. The index $i$ runs over every distinct interface surface $A_{i}$ and $\vec{D}=\epsilon_{0} \epsilon_{r} \vec{E}$ corresponds to the displacement field.

On the other hand, the photoelastic contribution to the optomechanical coupling occurs due to the strain field $(S)$ modulation of the dielectric properties, i.e., $\Delta\left(\frac{1}{\epsilon}\right)_{i j}=p_{i j k l} S_{k l}$ [29]. Only three different components for $p_{i j k l}$ are nonzero, due to the cubic symmetry [31]. Since the nondiagonal components of the strain $S_{k l}$ for the pillars are nonzero, in principle also $p_{44}$ should be taken into account [32]. However, because of the prevalent $z$-polarized character of the acoustic modes [9], it turns out that the error induced by considering only the $p_{12}$ contribution to $g_{0}^{\mathrm{ph}}$ is around $0.1 \%$. Thus, we only consider $p_{12} \sim 0.702$ from Ref. [27], a value corresponding to room temperature, and $\sim 880 \mathrm{~nm}$. Again, we consider $p_{12}$ to be nonvanishing only in the GaAs spacer layer. The photoelastic coupling contribution is then approximately given by

$$
g_{0}^{\mathrm{ph}}=\frac{\omega_{\mathrm{opt}} \epsilon_{0}}{2} \frac{\int n^{4} p_{12} S_{z}|\vec{E}|^{2} d V}{\int \epsilon|\vec{E}|^{2} d V} x_{\mathrm{ZPF}},
$$

where $n=n(\vec{r})$ is the refractive index and $S_{z}=\frac{\partial U_{z}}{\partial z}$. Note the strong similarity of this expression to the pump and probe detection efficiency given by Eq. (11).

For computational efficiency, all finite-element simulations have been performed for a cylindrical symmetry, i.e., for circular-section pillars. While the exact mode distribution changes between a square and a cylindrical pillar geometry, the overall dependence on lateral size should not change in more than a constant, as evidenced in Eq. (14). In Fig. 7 we present $g_{0}^{\text {geo }}$ (right scale) and $g_{0}^{\text {ph }}$ (left scale) as a function of the diameter $D$. By reducing the diameter from $10 \mu \mathrm{m}$ to 1 $\mu \mathrm{m}, g_{0}^{\text {geo }}$ increases from $\sim 6 \mathrm{kHz}$ to $\sim 40 \mathrm{kHz}$. Although the dependence of $g_{0}^{\mathrm{ph}}$ is similar to that of $g_{0}^{\text {geo }}$, as expected for GaAs [29,33], $\sim 10 \mathrm{~nm}$ below the resonance the magnitudes are more than 10 times larger, reaching values from $\sim 80$ to $\sim 550 \mathrm{kHz}$. In the inset of Fig. 7 we plot both contributions as a function of $1 / D$. Note the linear dependence on the inverse of the diameter, in agreement with the simpler conceptual model prediction given by Eq. (18). Interestingly, the 


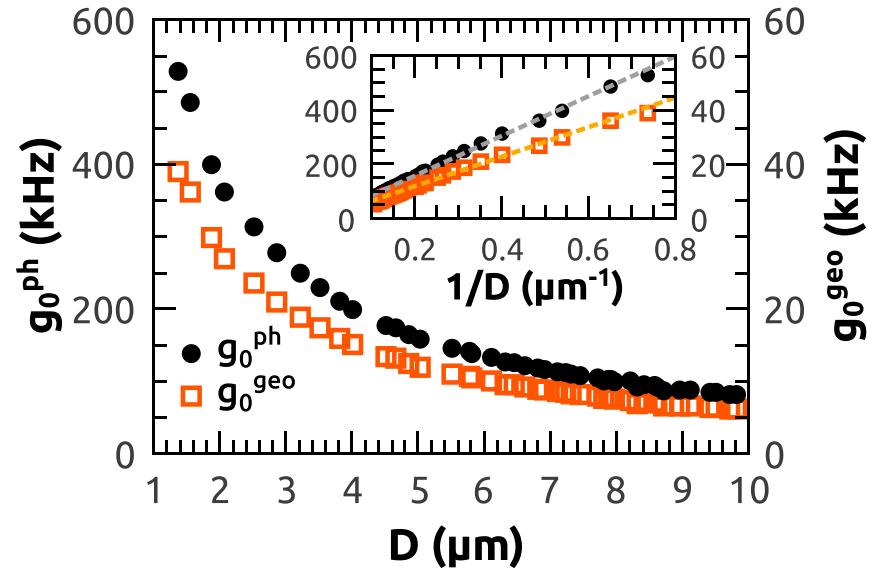

FIG. 7. Geometric ( $g_{0}^{\text {geo }}$, right scale) and photoelastic $\left(g_{0}^{\text {ph }}\right.$, left scale) single-photon optomechanical coupling rates as a function of pillar diameter $D$. The inset shows the $1 / D$ dependence for both contributions. Here $g_{0}^{\text {ph }}$ is more than an order of magnitude stronger than $g_{0}^{\text {geo }}$ for all calculated diameters. The dashed lines in the inset are linear fits.

results reported in Ref. [27] also imply that several order of magnitude added enhancement in $g_{0}^{\mathrm{ph}}$ should be accessible by reducing the operating temperature of the device to a few $\mathrm{K}$ [21]. This prediction, however, remains to be experimentally demonstrated.

Our results show that maximal optomechanical interactions are expected when the size of the micropillar is reduced. At the same time, as we have demonstrated in Fig. 5, a reduction of $L$ can affect the mechanical (and also optical) performance of the device. All these parameters are relevant to determine the applicability of the pillars as cavity optomechanical devices. Most studied cavity optomechanical systems operate with values of $\frac{g_{0}}{\kappa}$ between $10^{-6}$ and $10^{-3}$, while single-photon cooperativities typically fall between $10^{-2}$ and $10^{-8}$ [11].

Under the conditions studied the micropillars fall at the lower boundary of these ranges. The largest value we found for the single-photon cooperativity $C_{0}$ corresponds to the $3-\mu \mathrm{m}$ pillar, with $C_{0} \approx 3.8 \times 10^{-9}$. Also, for this particular size $\frac{g_{0}}{\kappa} \approx 1.3 \times 10^{-6}$. The micropillars studied have optical quality factors $Q_{\text {opt }}$ that range from $\sim 1.3 \times 10^{4}$ for pillars with $L \geqslant 5 \mu \mathrm{m}$ to $\sim 5 \times 10^{3}$ for $L=2 \mu \mathrm{m}$. These values can be pushed at least an order of magnitude, up to $\sim 10^{5}$, with the same fabrication technology [34]. On the other hand, the measured mechanical quality factors $Q_{m}$ fall in the range of $10^{3}$ and could in principle be pushed up to $\sim 10^{5}$ by addressing the issue of the surface-induced decay evidenced by the results in Fig. 5 and reducing the temperature (thus limiting anharmonic decay). A noteworthy point regarding this issue is that, even for close-to-ideal surfaces, it is known that surface dissipation through two-level systems can be an important mechanism that limits $Q_{m}$ [35]. In the case that the latter becomes the dominant path for dissipation of mechanical vibrations, and since it is based not on scattering but on absorption [35], the dependence of the mechanical lifetimes on lateral size is expected to deviate from that shown in Fig. 5.

Using both the calculated values for $g_{0}$ and the optimized values for $Q_{m}$ and $Q_{\mathrm{opt}}$, we expect that the maximum value for these parameters in the pillars can reach $\frac{g_{0}}{\kappa} \gtrsim 10^{-5}$ and $C_{0} \gtrsim$ $10^{-6}$. Moreover, were it possible to increase $g_{0}$, the attainable values for both $C_{0}$ and $\frac{g_{0}}{\kappa}$ would be greatly improved. It will be of great relevance, therefore, to experimentally confirm the huge efficiencies predicted at low temperatures and at resonance due to the resonant nature of photoelastic interactions [21]. Also the role of potentially stronger thermoelastic and optoelectronic forces should be investigated in the context of optical resonant excitation close to the direct gap absorption in these semiconductor materials. In any case, besides the mentioned optomechanical applications, it is clear that the main assets of the micropillars are their high operation frequencies and the possible integration with optoelectronic degrees of freedom; for example, they are particularly fit for experiments where one could link the vibrations to a single quantum dot, which could in principle be all quantum at temperatures below $4 \mathrm{~K}$. For such low-temperature applications, however, heating of the sample due to laser excitation may become an issue $[36,37]$. For the powers used in the experiments we report here ( $5 \mathrm{~mW}$ for the pump pulses, $0.5 \mathrm{~mW}$ for the probe) an increase of $\sim 1.5 \mathrm{~K}$ at room temperature has been estimated.

\section{CONCLUSION}

We presented detailed differential microreflectivity experiments and an effective model describing the mechanical behavior of a micropillar cavity. We derived simple scaling rules for the global mechanical and optomechanical trends of the system, which reproduce the pump-probe experiments performed over a series of different lateral sizes. With the presented model, we have satisfactorily explained the lateral confinement-induced mechanical mode frequency shift, the change in the modes lifetimes, and the increase of the optomechanical coupling.

As expected from the optical domain [16], the resonance frequencies of the confined acoustic modes depend on the lateral dimensions of the micropillars. Namely, their values increase when the size is reduced. We have shown, however, that this trend is frequency dependent, i.e., higher-frequency modes are less affected than the fundamental mechanical resonance. It was also observed that the mechanical quality factor increases with the lateral micropillar size, evidencing the detrimental effect of the surface defects on the lifetime of the cavity-confined phonons. Because of the frequency dependence of the in-plane effective speed of these modes, the effect turns out to be more critical for the lower-energy vibrations.

The increase of the optomechanical coupling strongly affects the detection efficiency in a differential reflectivity experiment. The measurements show a clear increase in the signal amplitude with decreasing lateral dimensions. By performing finite-element simulations, we provided a quantitative estimate of the photoelastic and geometric contributions to the vacuum optomechanical coupling factor $g_{0}$, accounting in addition for the observed experimental trend.

We expect that the demonstrated general scaling properties of the relevant parameters will allow for the optimized design of micropillar resonators for optomechanical applications. 


\section{ACKNOWLEDGMENTS}

This work was partially supported by ANPCyT Grants PICT 2012-1661 and 2013-2047, the Labex NanoSaclay, the
International Franco-Argentinean Laboratory LIFAN (CNRSCONICET), the French Agence Nationale pour la Recherche (grant ANR QDOM), the French RENATECH network, and ERC Starting Grant No. 715939 NanoPhennec.
[1] L. Ding, C. Baker, P. Senellart, A. Lemaître, S. Ducci, G. Leo, and I. Favero, Phys. Rev. Lett. 105, 263903 (2010).

[2] X. Sun, X. Zhang, and H. X. Tang, Appl. Phys. Lett. 100, 173116 (2012).

[3] R. Van Laer, B. Kuyken, D. Van Thourhout, and R. Baets, Nat. Photon. 9, 199 (2015).

[4] R. A. Norte, J. P. Moura, and S. Gröblacher, Phys. Rev. Lett. 116, 147202 (2016).

[5] A. G. Krause, M. Winger, T. D. Blasius, Q. Lin, and O. Painter, Nat. Photon. 6, 768 (2012).

[6] Y. Imade, R. Ulbricht, M. Tomoda, O. Matsuda, G. Seniutinas, S. Juodkazis, and O. B. Wright, Nano Lett. 17, 6684 (2017).

[7] S. F. Preble, Q. Xu, B. S. Schmidt, and M. Lipson, Opt. Lett. 30, 2891 (2005)

[8] S. Anguiano, A. E. Bruchhausen, B. Jusserand, I. Favero, F. R. Lamberti, L. Lanco, I. Sagnes, A. Lemaître, N. D. LanzillottiKimura, P. Senellart, and A. Fainstein, Phys. Rev. Lett. 118, 263901 (2017).

[9] F. R. Lamberti, Q. Yao, L. Lanco, D. T. Nguyen, M. Esmann, A. Fainstein, P. Sesin, S. Anguiano, V. Villafañe, A. Bruchhausen, P. Senellart, I. Favero, and N. D. Lanzillotti-Kimura, Opt. Express 25, 24437 (2017).

[10] S. Anguiano, A. E. Bruchhausen, I. Favero, I. Sagnes, A. Lemaître, N. D. Lanzillotti-Kimura, and A. Fainstein, Phys. Rev. A 98, 013816 (2018).

[11] M. Aspelmeyer, T. J. Kippenberg, and F. Marquardt, Rev. Mod. Phys. 86, 1391 (2014).

[12] M. Ludwig, B. Kubala, and F. Marquardt, New J. Phys. 10, 095013 (2008).

[13] K. W. Murch, K. L. Moore, S. Gupta, and D. M. Stamper-Kurn, Nat. Phys. 4, 561 (2008).

[14] S. Weis, R. Rivière, S. Deléglise, E. Gavartin, O. Arcizet, A. Schliesser, and T. J. Kippenberg, Science 330, 1520 (2010).

[15] M. Yuan, V. Singh, Y. M. Blanter, and G. A. Steele, Nat. Commun. 6, 8491 (2015).

[16] J. P. Reithmaier, M. Röhner, H. Zull, F. Schäfer, A. Forchel, P. A. Knipp, and T. L. Reinecke, Phys. Rev. Lett. 78, 378 (1997).

[17] C. Reinhardt, R. Brückner, M. Sudzius, and S. I. Hintschich, Appl. Phys. Lett. 100, 103306 (2012).

[18] T. Gutbrod, M. Bayer, A. Forchel, P. A. Knipp, T. L. Reinecke, A. Tartakovskii, V. D. Kulakovskii, N. A. Gippius, and S. G. Tikhodeev, Phys. Rev. B 59, 2223 (1999).
[19] C. Thomsen, H. T. Grahn, H. J. Maris, and J. Tauc, Phys. Rev. B 34, 4129 (1986).

[20] A. Bartels, T. Dekorsy, H. Kurz, and K. Köhler, Phys. Rev. Lett. 82, 1044 (1999).

[21] A. Fainstein, N. D. Lanzillotti-Kimura, B. Jusserand, and B. Perrin, Phys. Rev. Lett. 110, 037403 (2013).

[22] J. M. Ziman, Electrons and Phonons: The Theory of Transport Phenomena in Solids (Oxford University Press, Oxford, 1960).

[23] A. R. Miller, R. M. Brown, and E. Vegh, IEE Proc. H 131, 114 (1984).

[24] A. A. Maznev, Phys. Rev. B 91, 134306 (2015).

[25] O. Matsuda, T. Tachizaki, T. Fukui, J. J. Baumberg, and O. B. Wright, Phys. Rev. B 71, 115330 (2005).

[26] M. F. Pascual-Winter, A. Fainstein, B. Jusserand, B. Perrin, and A. Lemaître, Phys. Rev. B 85, 235443 (2012).

[27] B. Jusserand, A. N. Poddubny, A. V. Poshakinskiy, A. Fainstein, and A. Lemaître, Phys. Rev. Lett. 115, 267402 (2015).

[28] D. J. Griffiths, Introduction to Electrodynamics, 3rd ed. (Dorling Kindersley, London, 2007).

[29] C. Baker, W. Hease, D.-T. Nguyen, A. Andronico, S. Ducci, G. Leo, and I. Favero, Opt. Express 22, 14072 (2014).

[30] S. G. Johnson, M. Ibanescu, M. A. Skorobogatiy, O. Weisberg, J. D. Joannopoulos, and Y. Fink, Phys. Rev. E 65, 066611 (2002).

[31] R. E. Newnham, Properties of Materials: Anisotropy, Symmetry, Structure (Oxford University Press, Oxford, 2005).

[32] O. Florez, P. F. Jarschel, Y. A. V. Espinel, C. M. B. Cordeiro, T. P. M. Alegre, G. S. Wiederhecker, and P. Dainese, Nat. Commun. 7, 11759 (2016).

[33] K. C. Balram, M. Davanço, J. Y. Lim, J. D. Song, and K. Srinivasan, Optica 1, 414 (2014).

[34] V. Loo, L. Lanco, A. Lemaître, I. Sagnes, O. Krebs, P. Voisin, and P. Senellart, Appl. Phys. Lett. 97, 241110 (2010).

[35] M. Hamoumi, P. E. Allain, W. Hease, E. Gil-Santos, L. Morgenroth, B. Gérard, A. Lemaître, G. Leo, and I. Favero, Phys. Rev. Lett. 120, 223601 (2018).

[36] S. M. Meenehan, J. D. Cohen, G. S. MacCabe, F. Marsili, M. D. Shaw, and O. Painter, Phys. Rev. X 5, 041002 (2015).

[37] B. D. Hauer, P. H. Kim, C. Dooling, F. Souris, and J. P. Davis, arXiv:1710.09439v3 [Phys. Rev. B (to be published)]. 